\title{
Estudo teórico sobre as propriedades eletrônicas de nanoestruturas de carbono e h-BN
}

\author{
Guilherme Angelo Moreira Bernardoํㅜㄹ Mirleide Dantas Lopes ${ }^{1} \&$ Sérgio André \\ Fontes Azevedo ${ }^{2}$
}

(1) Universidade Federal de Campina Grande, Centro de Formação de Professores, Unidade Acadêmica de Ciências Exatas e da Natureza, Rua Sérgio Moreira de Figueiredo, Casas Populares, Cajazeiras 58900000, Paraíba, Brasil. E-mail: guilhermesa1996@hotmail.com, mirleide_dantas@yahoo.com.br

(2) Universidade Federal da Paraíba - Campus I, Centro de Ciências Exatas e da Natureza, Departamento de Física, Programa de Pós-Graduação em Física, Cidade Universitária, Castelo Branco 58051-900, João

Pessoa, Paraíba, Brasil. E-mail: sazevedo@fisica.ufpb.br

Bernardo G.A.M., Lopes M.D. \& Azevedo S.A.F. (2018) Estudo teórico sobre as propriedades eletrônicas de nanoestruturas de carbono e h-BN. Pesquisa e Ensino em Ciências Exatas e da Natureza, 2(1): 71-81. http://dx.doi.org/10.29215/pecen.v2i1.588

Resumo: O estudo dos nanomateriais tem se consolidado como uma nova revolução no meio científico. Dentre as nanoestruturas mais investigadas atualmente encontram-se as derivadas do carbono e do nitreto de boro hexagonal (h-BN). O grafeno, por exemplo, trata-se de uma rede bidimensional hexagonal, formada exclusivamente por átomos de carbono. Neste sentido, investigamos a energia de formação e a estrutura eletrônica de um plano de grafeno, um plano de h-BN, bem como, quatro nanocones, com disclinação de $60^{\circ}$ cada, sendo dois deles derivados do grafeno e dois derivados do h-BN. Tais investigações foram feitas por meio do Código SIESTA, um software livre que utiliza métodos de primeiros princípios, baseados na Teoria do Funcional da Densidade (DFT), como parâmetro para sua execução. A partir das observações realizadas foi possível identificar quais nanoestruturas apresentaram maior estabilidade, a depender da organização atômica de suas respectivas redes cristalinas. Nos nanomateriais investigados percebemos que, tanto a energia de formação por átomo, quanto à densidade eletrônica, variaram sensivelmente em função dos diferentes arranjos atômicos. Com relação à condutividade elétrica, as estruturas pesquisadas apresentaram boa concordância com a literatura, reafirmando assim a aplicabilidade destes nanomateriais em dispositivos eletrônicos.

Palavras chave: Nanomateriais, energia de formação, densidade eletrônica, estabilidade.

\section{Theoretical study on the electronic properties of carbon nanostructures and h-BN}

Abstract: The study of nanomaterials has consolidated as a new revolution in the scientific environment. Among the most investigated nanostructures currently are the derivatives of carbon and hexagonal boron nitride (h-BN). Graphene for example it is a hexagonal two-dimensional lattice formed only by carbon atoms. In this sense, we investigated the formation energy and the electronic structure of a graphene plane, a h-BN plane, as well as, four nanocones, with disclination of $60^{\circ}$ each, two of them derived from graphene and two derived from h-BN. These investigations were made through the SIESTA Code, a free software that uses first-principles methods, based on Density Functional Theory (DFT), as a parameter for its execution. From observations it was possible to identify which nanostructures showed greater stability, depending on the atomic organization of their respective crystal lattices. In the investigated nanomaterials we realized that both the formation energy per atom and the electron density varied noticeably in function of the different atomic arrangements. Regarding the electrical conductivity, the structures researched presented good agreement with the literature, thus reaffirming the applicability of these nanomaterials in electronic devices.

Key words: Nanomaterials, formation energy, electronic density, stability. 


\section{Introdução}

Para refletirmos sobre os inúmeros avanços científicos e tecnológicos que hoje são experimentados, devemos partir da compreensão de que a construção do atual estágio de desenvolvimento não foi linear e sim concebida a partir de inúmeras rupturas e revoluções. Desde que saímos da produção de ferramentas de pedra, no paleolítico, até a fabricação dos modernos lasers cirúrgicos, da construção dos grandes relógios de corda das catedrais na Idade Média, a relógios atômicos utilizados em satélites de navegação por GPS, muita coisa mudou no modo como vemos e fazemos Ciência (Schulz 2005).

Essas descontinuidades são concebidas por intermédio das necessidades vivenciadas pelas sociedades em determinados períodos históricos. Neste ínterim, podemos citar, por exemplo, a relação entre a Física Clássica, organizada até o final do século XIX, e a Física Moderna. A primeira, ocupa-se especialmente do estudo dos fenômenos naturais no mundo macroscópico. A segunda, descreve o comportamento e as interações que acontecem a nível atômico e molecular, bem como os corpos que podem mover-se com velocidades próximas a da luz. Tal categorização parte de um longo processo de aprimoramento científico.

Em meio a esse cenário de rupturas, nos dias atuais podemos citar a Nanociência e Nanotecnologia (N\&N), que apresenta-se, assim como a máquina a vapor e a eletricidade, como uma revolução contemporânea nos meios de produção do conhecimento científico e tecnológico (Hett 2004).

Nesse sentido, a Nanociência é entendida como a área de conhecimento que parte da interpretação e compreensão dos princípios fundamentais de moléculas e arranjos atômicos, desde que compreendidas entre 1 e 100 nanômetros. Estes arranjos moleculares são denominados nanomateriais. De modo complementar, a Nanotecnologia é a manipulação desses nanomateriais com vistas em aplicações tecnológicas (Alves 2004).

A N\&N surge em meio a um contexto de miniaturização. No entanto, as dimensões relativas a essa escala são muito pequenas, dado que um nanômetro é a bilionésima parte do metro $(10-9 \mathrm{~m})$. Logo, as interações e fenômenos observados nesta ordem de grandeza não podem ser completamente descritos por leis científicas do mundo macroscópico, como é o caso da Mecânica Clássica, sendo necessária a utilização de outros formalismos, em especial, o da Mecânica Quântica.

As primeiras indagações visionárias feitas acerca da manipulação da matéria a nível atômico e molecular, e de seus benefícios à sociedade, foi proposta pelo físico americano Richard Feynman, em uma palestra proferida no encontro anual da Sociedade Americana de Física (APS), no Instituto de Tecnologia da Califórnia (CALTECH), em 29 de dezembro de 1959, intitulada "Há mais espaço lá embaixo" ("There is plenty of Room at the Bottom"). Na ocasião, Feynman fez reflexões intrigantes sobre um mundo que ele chamou de "surpreendentemente pequeno". Ele supôs, por exemplo, que a manipulação de arranjos moleculares tornaria possível a escrita de 24 volumes inteiros da Enciclopédia Britânica na cabeça de um alfinete. A palestra de Feynman é certamente o marco de uma nova era, que podemos chamar de revolução nano (Feynman 1959).

A N\&N é uma nova forma de pensar a ciência e a tecnologia, seus avanços trarão benefícios para a vida moderna, como também possíveis malefícios. Devemos estar preparados para compreender tais modificações e prontos para atualizar-se nos progressos vindouros. A ascensão é rápida, o nanomundo promete enormes rupturas e os nanomateriais anunciam uma verdadeira corrida do ouro (“Gold Rush”) (Geim \& Novoselov 2007).

Desta forma, é de fundamental importância compreendermos como tem se consolidado o estudo dos materiais na escala nanométrica e suas inúmeras aplicações nas mais diversas áreas do conhecimento, pois, assim sendo, estaremos mais familiarizados com as pesquisas desenvolvidas na contemporaneidade, bem como, aptos a contribuir com o desenvolvimento científico que se estabelece.

Diversas áreas do conhecimento humano têm se dedicado ao estudo dos nanomateriais, com vistas a buscar meios de promover o bem-estar social e fortalecer os vínculos 
interdisciplinares para o progresso da ciência. Nesse sentido, a Física, mais especificamente a Física da Matéria Condensada, tem se ocupado em investigar as inúmeras características desses materiais, tais como: propriedades eletrônicas, térmicas e óticas; especialmente com intuito de avaliar a empregabilidade destes na produção de dispositivos eletrônicos.

Nesta perspectiva, o presente artigo traz uma análise sobre a possível aplicabilidade de alguns nanomateriais, constituídos basicamente por carbono, nitrogênio e boro, com o intuito de verificar a possível aplicação destes em dispositivos eletrônicos, a partir de suas características estruturais e eletrônicas.

\section{Materiais e Métodos}

$\mathrm{Na}$ investigação teórica de nanomateriais é comum a utilização de simulações computacionais. Um dos meios de se estudar as propriedades de um dado material é utilizando o Código SIESTA, um software livre que permite obter, a partir de simulações, o comportamento de estruturas que compõem a matéria (Soler et al. 2002), software este que foi utilizado na presente investigação.

O SIESTA baseia-se em dados fornecidos sobre a estrutura dos nanomateriais, como por exemplo, quais e quantos átomos a compõem, bem como as coordenadas espaciais atômicas da rede cristalina (Kittel 2013). O programa nos retorna informações sobre a energia da estrutura, densidade eletrônica, momento de dipolo elétrico, dentre outras. A possibilidade de tratar grandes sistemas dá ao SIESTA um ótimo padrão de eficiência no estudo dos nanomateriais.

Este software utiliza a Teoria do Funcional da Densidade (DFT) como base para sua execução (Soler et al. 2002). A referida teoria foi proposta em um artigo publicado em 1964 por Walter Kohn e seu aluno Pierre Hohenberg. A DFT consiste em uma reformulação na forma de encontrar a energia, a partir da equação de Schrödinger, proposta pela mecânica quântica. Enquanto esta baseia-se em funções de onda, para o cálculo da energia, aquela faz uso da densidade eletrônica, como parâmetro na observação dos fenômenos que envolvem a previsão das propriedades dos átomos, moléculas e estruturas cristalinas, o que reduz significativamente os cálculos (Hohenberg \& Kohn 1964).

A proposição prática para a DFT veio um ano depois, em um artigo publicado por Kohn e Lu Sham (Kohn \& Sham 1965). Juntos, esses dois artigos formam a base da teoria. Com os adventos tecnológicos e a utilização das equações de Kohn-Sham, a resolução de problemas complexos pôde ser facilitada, significando um novo ciclo nos métodos utilizados no estudo das propriedades da matéria.

\section{Estruturas investigadas}

Dentre os nanomateriais mais estudados na atualidade, encontram-se os derivados do carbono. Isso é justificável pelo fato de que o carbono é um dos elementos químicos mais abundantes na natureza e apresenta uma distribuição eletrônica muito peculiar, $1 \mathrm{~s}^{2} 2 \mathrm{~s}^{2} 2 \mathrm{p}^{2}$, que o torna tetravalente, favorecendo a sua associação com diversos outros átomos.

Em meio aos inúmeros alótropos do carbono, encontra-se o grafeno (Figura 1). Este por sua vez é uma folha da espessura de único átomo, composta exclusivamente por carbono, que se arranjam em hexágonos. Porém as asserções científicas sobre a estabilidade de redes cristalinas bidimensionais inferia que esses arranjos periódicos eram termodinamicamente instáveis e não poderiam ser produzidos ou isolados (Landau 1937).

Por outro lado, em 2004, um grupo de cientistas da Universidade de Manchester, na Inglaterra, e do Instituto de Tecnologia Microeletrônica, na Rússia, coordenados por Konstantin Novoselov e Andre Geim, conseguiram isolar e descrever filmes de grafite monocristalinos de poucos átomos de espessura (incluindo o grafeno), mas que não deixam de ser estáveis em condições ambientes (Novoselov et al. 2004). A sintetização do grafeno é sem dúvidas um marco para a ciência moderna. 


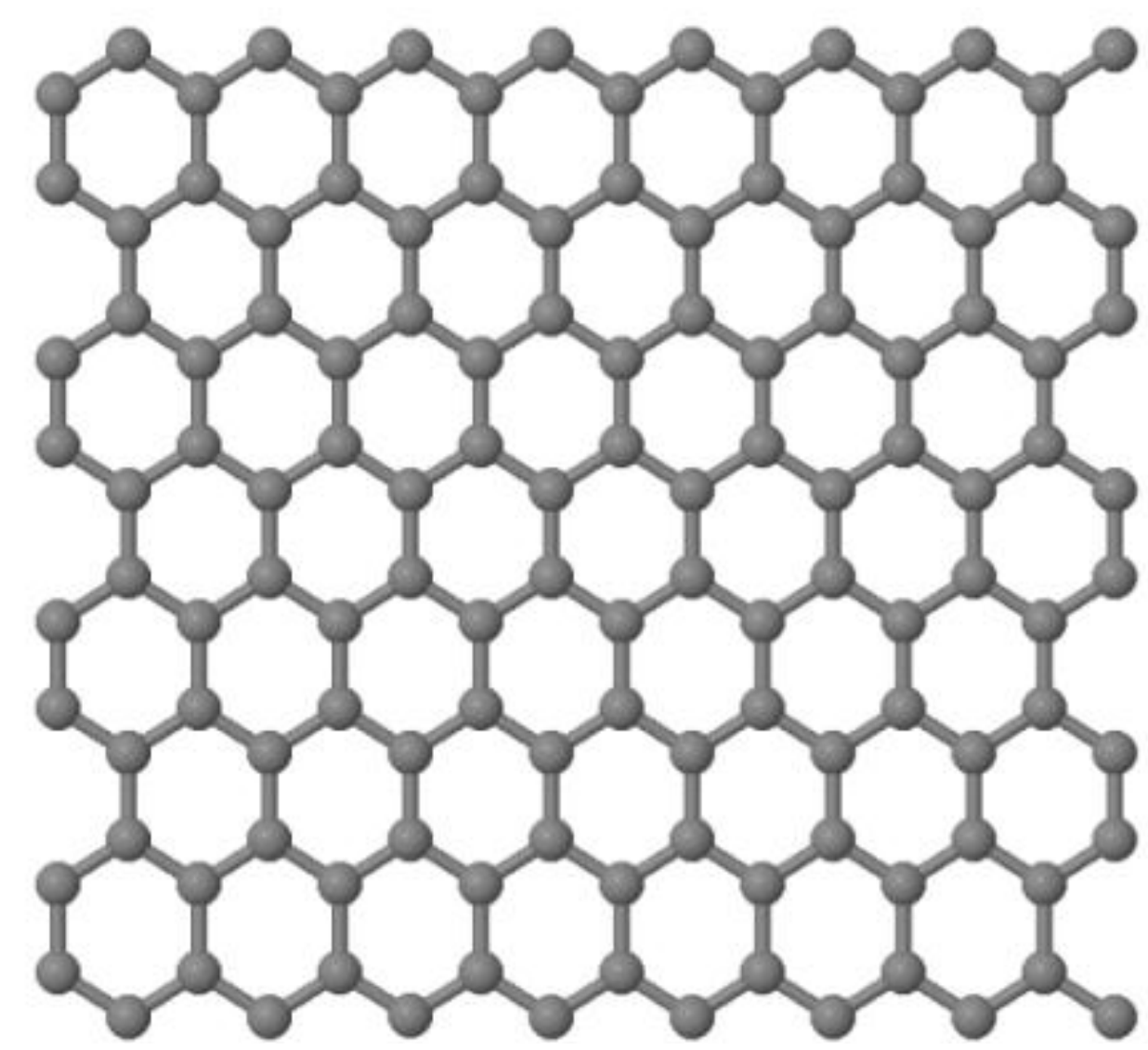

Figura 1. Plano de grafeno formado exclusivamente por átomos de carbono.

O grafeno exibe propriedades mecânicas, térmicas, químicas e eletrônicas bastante singulares (Cano-Marquez et al. 2015). As ligações de carbono neste plano apresentam hibridização $\mathrm{sp}^{2}$, ligações fortes que garantem a estabilidade da estrutura. No entanto, perpendicular ao plano, há um orbital pz semipreenchido, que favorece a mobilidade dos elétrons na rede (Klein et al. 2001).

Esse nanomaterial pode ser modelado de várias maneiras, formando, por exemplo, nanotubos de carbono, a partir do "enrolamento" da nanofolha, ou arranjando-se em nanocones (Figura 2), por meio de "cortes e junções" no plano, um processo denominado de Volterra. Esse método de "cortar e colar" define o ângulo de abertura do nanocone que se deseja obter (Pedreira 2011).

A princípio o grafeno é plano, no entanto, sua curvatura pode ser modificada a depender da disclinação efetuada, ou seja, a depender do setor $\pi / 3$ inserido ou retirado da estrutura. Desta forma, se retirarmos uma região $\pi / 3$ do plano, estaremos inserindo uma disclinação positiva de $60^{\circ}$ e formaremos um cone com um pentágono no vértice. Se ao invés de retirarmos, inserirmos um setor $\pi / 3$, estaremos formando uma disclinação negativa de $60^{\circ}$ e aparecerá um heptágono na estrutura (Lopes et al. 2014). Dependendo da quantidade de setores $\pi / 3$ retirados, diferentes nanocones podem ser obtidos, porém, neste estudo, todos os nanocones investigados possuem disclinação positiva de $60^{\circ}$.

Os nanocones de carbono foram observados pela primeira vez em 1992, no Japão, fechando nanotubos (Iijima et al. 1992). Dois anos mais tarde, os nanocones puderam ser observados isoladamente (Ge \& Sattler 1994). Desde então inúmeros trabalhos sobre essas nanoestruturas foram desenvolvidos, em sua maioria, voltados para a possível utilização destes nanomateriais em dispositivos eletrônicos, o que certamente revolucionará a eletrônica que se conhece atualmente. Um fato peculiar é que, apesar dessas nanoestruturas terem sido observadas pela primeira vez fechando nanotubos, pouco tem sido discutido no âmbito teórico, neste sentido. 


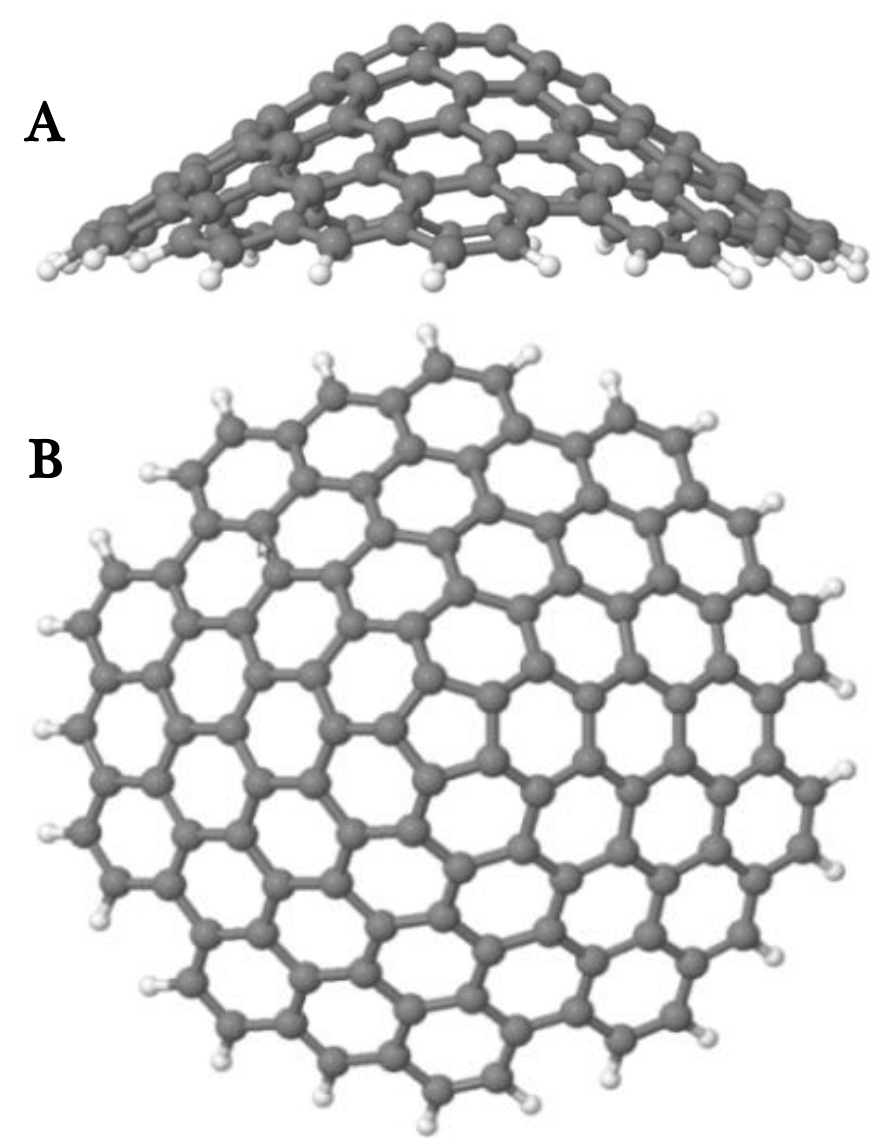

Figura 2. Nanocone de grafeno com saturação de hidrogênio nas pontas: A. Visão lateral e B. Visão frontal.

Além das nanoestruturas formadas por átomos de carbono, inúmeros arranjos moleculares têm sido estudados. Em meio a este cenário, encontram-se os nanomateriais formados por nitreto de boro hexagonal, também conhecidos por h-BN, e que são representados na Figura 3. Sua estrutura é análoga a do grafeno, no entanto é composta por dois átomos diferentes (Rubio et al. 1994). O h-BN pode ser obtido por deposição química a vapor, em uma combinação de óxido de boro e nitrogênio em suspensão, aliado a altas temperaturas (Han et al. 1998).

A partir do plano de h-BN também é possível construir outras nanoestruturas, a exemplo dos nanocones. Além disso, em se tratando deste material, a depender das condições do ambiente químico de sintetização, um cone com disclinação de $60^{\circ}$, pode apresentar características distintas, ligações B-B na junção (BNB) ou N-N (BNN), conforme ilustrados na Figura 4A-B, respectivamente.

Nesse sentido, nossa investigação buscou analisar a energia de formação e a densidade de estados de um plano de grafeno e um plano de h-BN, assim como suas respectivas estruturas cônicas em diferentes arranjos atômicos. A análise destas variáveis permite-nos obter informações sobre a estabilidade estrutural dos nanomateriais investigados, bem como inferir conclusões a respeito da condutividade elétrica destes.

As duas estruturas planas estudadas possuem 128 átomos. No caso do grafeno, obviamente, todos os átomos são de carbono. Em se tratando do h-BN, foram 64 boros e 64 nitrogênios. Já os nanocones foram analisados em situações diferentes. Foram investigados dois cones de carbono, um com 140 átomos (115 carbonos e 25 hidrogênios) e o outro com 200 átomos (170 carbonos e 30 hidrogênios). Assim foi possível realizarmos uma análise comparativa, quanto ao tamanho, entre essas duas nanoestruturas. Os nanocones de h-BN, apesentavam o mesmo número de átomos, no entanto, eles possuíam de diferentes arranjos atômicos de boro e nitrogênio; BNB, com 88 boros, 82 nitrogênios e 30 hidrogênios; e BNN, com 82 boros, 88 nitrogênios e 30 hidrogênios. 


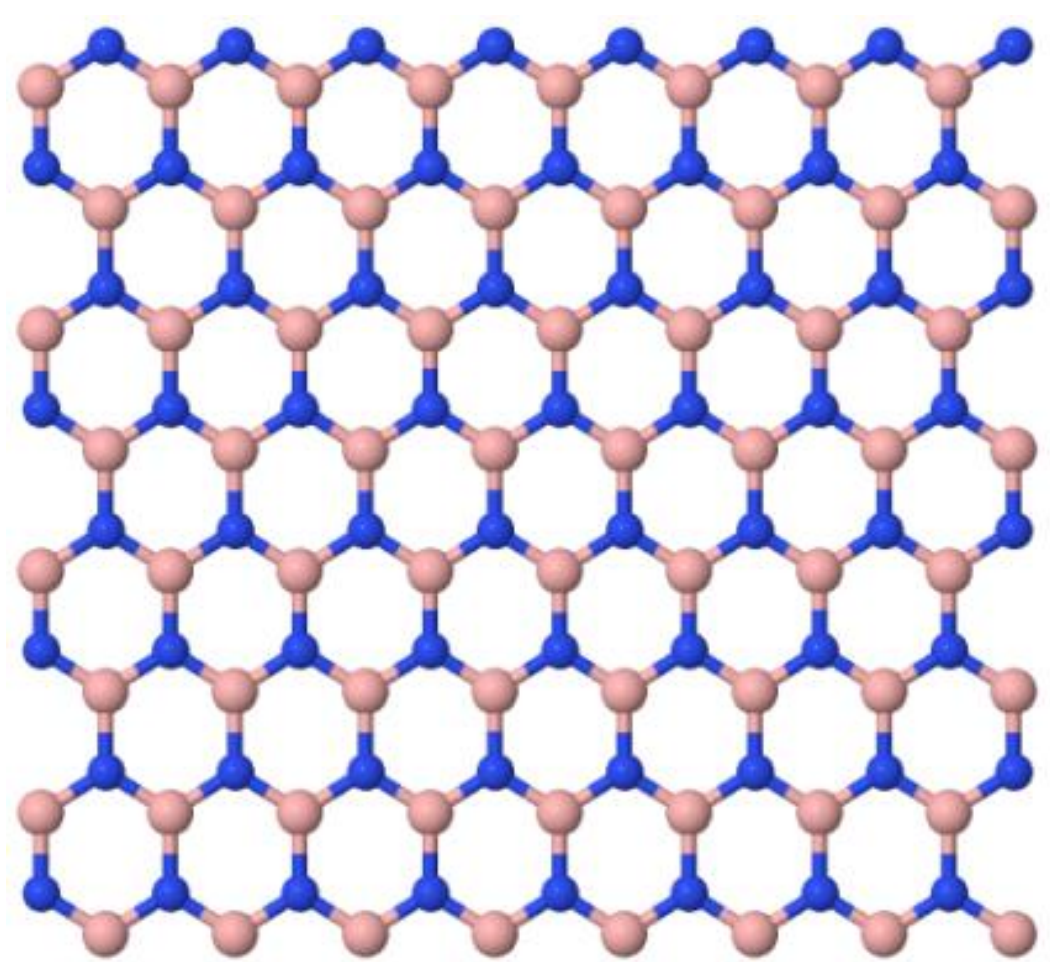

Figura 3. Plano de h-BN mostrando em rosa átomos de boro e em azul átomos de nitrogênio.
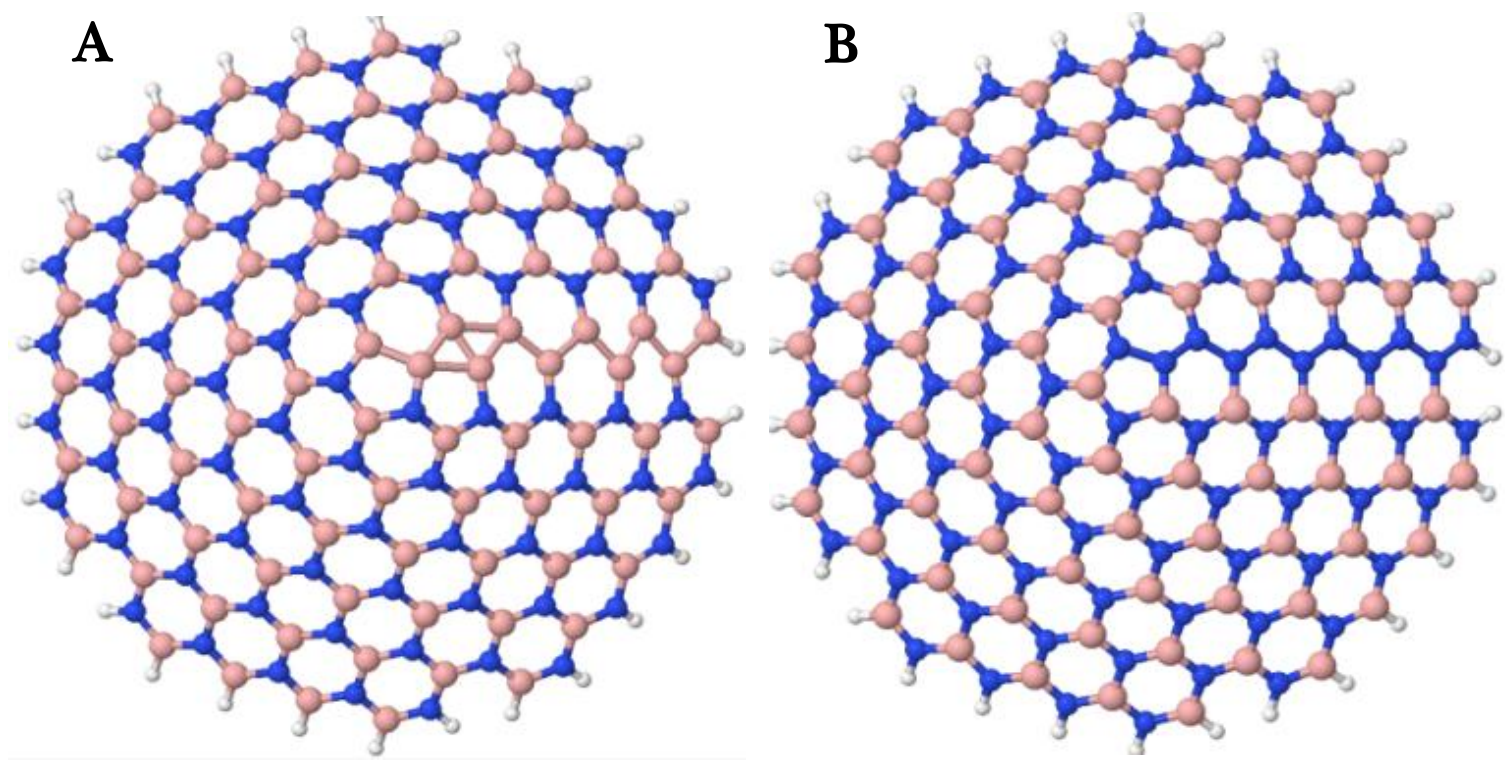

Figura 4. Nanocones de h-BN com saturação de hidrogênio nas pontas: A. Junção B-B (BNB) e B. Junção N$\mathrm{N}(\mathrm{BNN})$.

O cálculo da energia de formação destas nanoestruturas foi feito por meio da equação (1), em que, $E_{f}$ é a energia de formação, $E_{t o t}$ é a energia total do sistema, obtida através do Código SIESTA, $n_{C}, n_{B}, n_{N}$ e $n_{H}$ são, respectivamente, o número de átomos de carbono, boro, nitrogênio e hidrogênio presentes na estrutura, e $\mu_{C}, \mu_{B}, \mu_{N}$ e $\mu_{H}$ são, nesta ordem, os potenciais químicos destes elementos, que apresentam os seguintes valores: $\mu_{C}=-145,13 \mathrm{eV}, \mu_{B}=-69,9 \mathrm{eV}, \mu_{N}$ $=-261,33 \mathrm{eV}$ e $\mu_{H}=-12,33 \mathrm{eV}$. Estas quantidades também foram obtidas através do Código SIESTA, por meio da convergência de um único átomo de cada espécie química.

$$
E_{f}=E_{\text {tot }}-n_{C} \mu_{C}-n_{B} \mu_{B}-n_{N} \mu_{N}-n_{H} \mu_{H}
$$


A fim de realizarmos uma análise comparativa, a energia de formação, obtida a partir da equação (1), em cada estrutura, foi divida pelo seu respectivo número de átomos, resultados que serão apresentados a seguir.

\section{Resultados e Discussão}

Os nanomateriais apresentados nas Figuras 1-4 já estão convergidos, isto significa dizer que apresentam suas respectivas configurações mais estáveis, fornecidas pelo código SIESTA. Neste sentido é interessante observar que no cone BNB (Figura 4A), surgiram após a convergência dois triângulos na estrutura que não haviam sido inseridos inicialmente. Este resultado dialoga com pesquisas recentes que mostraram que estruturas planas feitas com átomos de boro são mais estáveis quando são constituídas por células triangulares e hexagonais (Tang \& Ismail-Beigi 2007).

A energia de formação por átomo, obtida para todos os nanomateriais aqui investigados, encontra-se listada na Tabela 1. A partir destes resultados é possível afirmar que a nanoestrutura que se mostrou mais estável foi o grafeno, uma vez que ele apresentou menor energia de formação por átomo (Azevedo et al. 2007). Através dos dados apresentados podemos inferir que o plano de grafeno é fisicamente mais estável do que o plano de h-BN. Isso se deve, especialmente, às ligações $\mathrm{C}-\mathrm{C}$, interações relativamente fortes, no plano. No entanto, ambos os nanomateriais são estáveis e apresentam pouca diferença em suas energias de formação por átomo, além de já terem sido obtidos experimentalmente.

Tabela 1. Energia de formação para os sistemas em eletrovolts por átomo (eV/atom).

\begin{tabular}{lcc}
\hline \multicolumn{1}{c}{ Estrutura } & Número de átomos & $E_{f}(\mathrm{eV}) /$ atom \\
\hline Plano Grafeno & 128 & $-10,24$ \\
Plano h-BN & 128 & $-9,05$ \\
Nanocone de Carbono 1 & 140 & $-8,25$ \\
Nanocone de Carbono 2 & 200 & $-8,43$ \\
Nanocone BNB & 200 & $-8,08$ \\
Nanocone BNN & 200 & $-8,10$ \\
\hline
\end{tabular}

Com relação aos nanocones de carbono, percebemos que quanto maior for o número de átomos, maior será a estabilidade da estrutura, pois existe um aumento no número de ligações C-C, que favorece o equilíbrio físico do nanomaterial (Azevedo 2006). Além disso, as interações no plano são covalentes, fato que aumenta a estabilidade da estrutura. Este resultado parece, de certo modo, intuitivo, pois quanto menor o número de átomos, menos energia será necessária para romper as ligações ou "quebrar" as nanoestruturas, no entanto, estamos considerando a energia de formação por átomo, o que implica em avaliar a energia necessária para cada átomo se estabilizar na estrutura.

Para os nanocones de h-BN, podemos observar que a estrutura do tipo BNN é mais estável do que a BNB, apesar de suas energias de formação por átomo apresentarem diferença mínima. Este resultado decorre do fato de as ligações N-N serem mais estáveis que as ligações BB (Dantas et al. 2015).

Quanto à densidade de estados, na Figura 5, apresentamos os resultados referentes ao grafeno e o h-BN e na Figura 6 os relativos aos diferentes nanocones. No caso do grafeno, observamos que há um número considerável de estados próximos à energia de Fermi, que se encontra transladada para origem do gráfico. Isto o caracteriza como um semicondutor, pois ao calcularmos o pequeno "gap" de energia entre os estados ocupados (estados de valência, antes do nível de Fermi) e os estados desocupados (estados de condução, depois do nível de Fermi), veremos que ele será da ordem de 0,5 eV (Rezende 2015).

Este dado nos mostra que, para o caso do grafeno, os estados de condução e de valência estão bem próximos, ou seja, ele apresenta um "gap" estreito. Tal observação se alinha aos resultados obtidos experimentalmente e viabiliza as propostas encontradas na literatura de que é 
possível controlar o "gap", por exemplo, com a variação do campo elétrico, possibilitando assim sua aplicação em dispositivos eletrônicos (Novoselov et al. 2004).
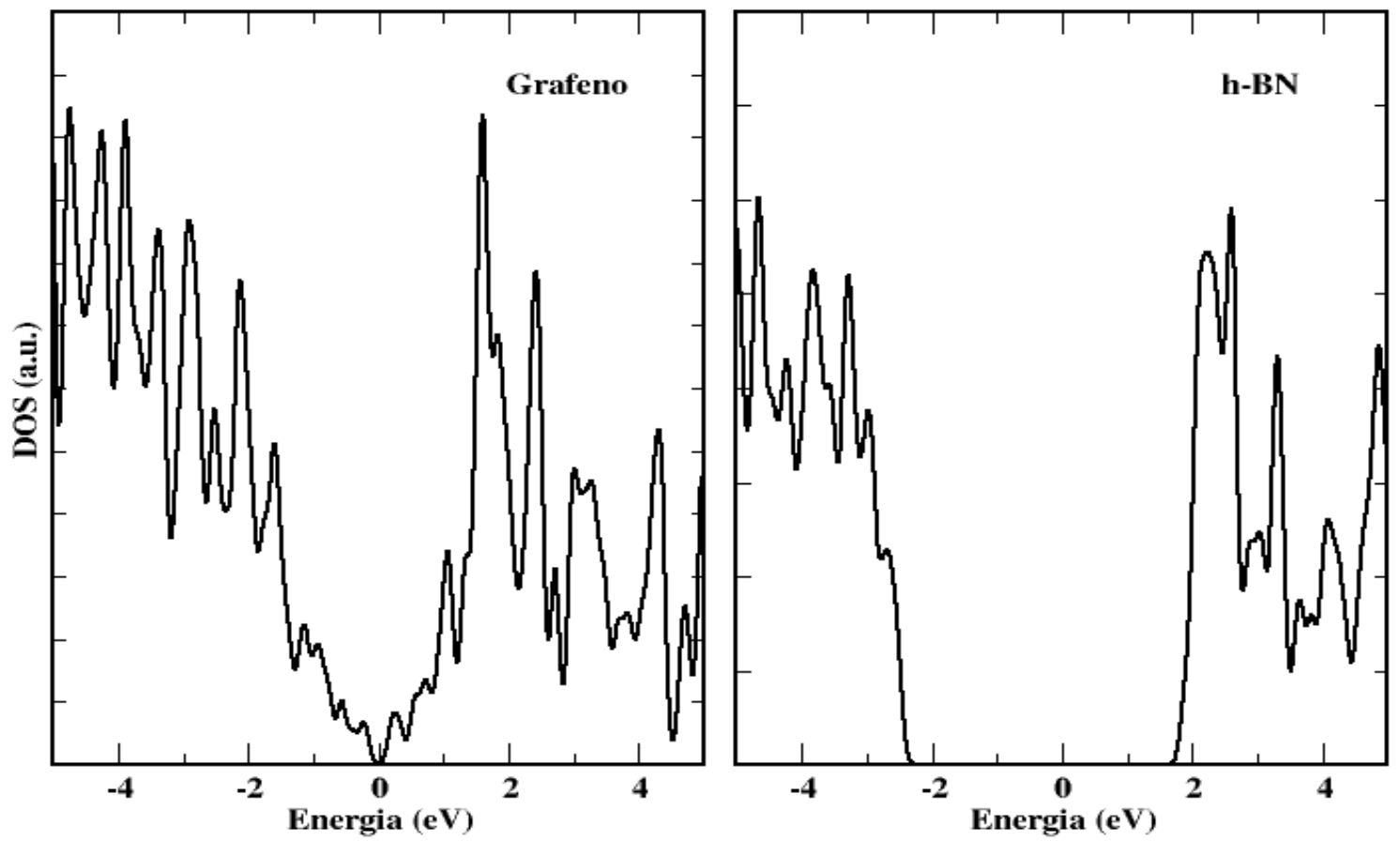

Figura 5. Comparação das densidades de estado do grafeno e do h-BN.
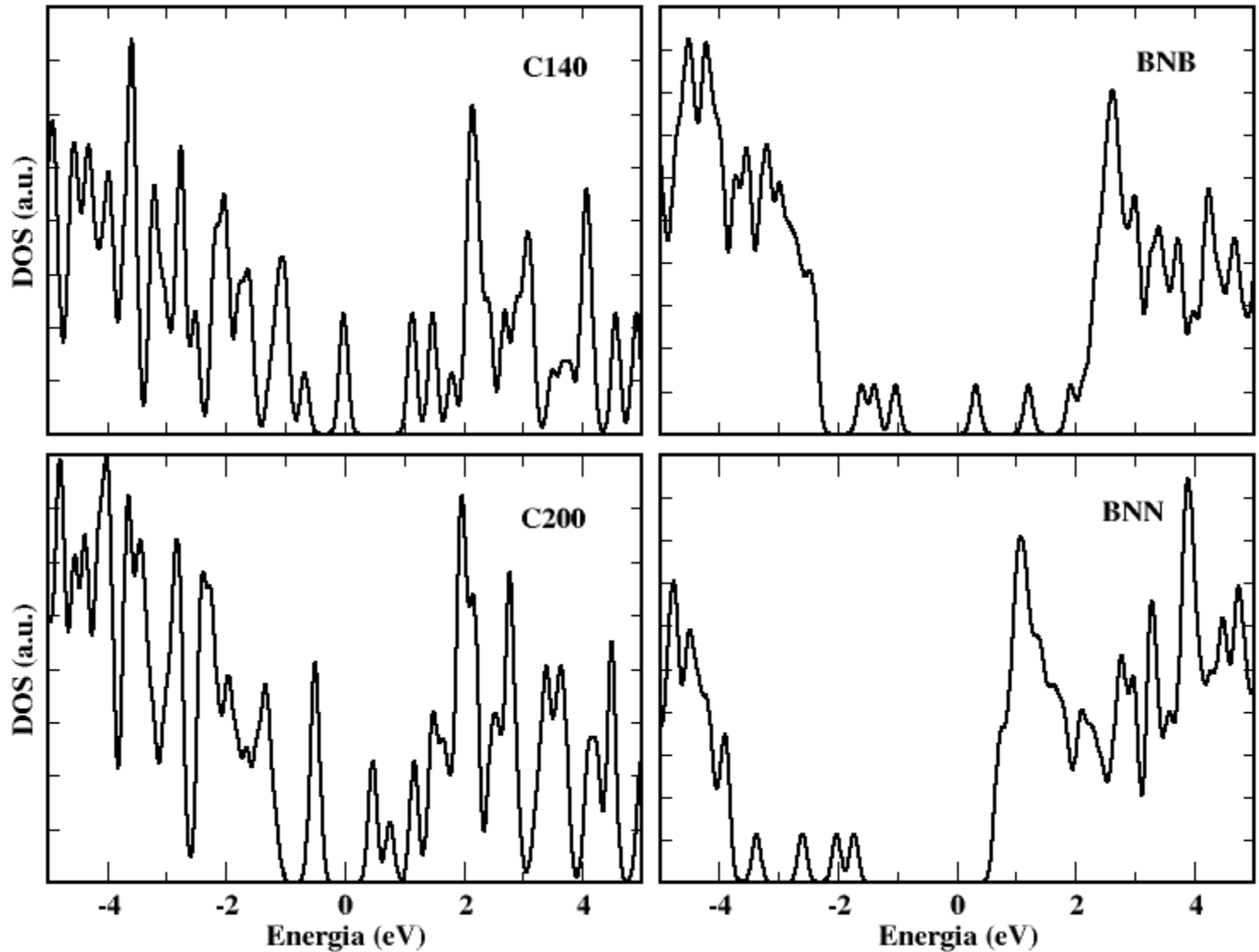

Figura 6. Comparação entre as densidades de estado dos nanocones de carbono e de h-BN (BNB e BNN). 
Observando o comportamento eletrônico do plano de h-BN, percebemos que sua densidade de estados apresenta caraterísticas típicas de um material isolante, isso é notado pela distância relativamente alta entre os estados preenchidos, valência, e os estados desocupados, condução. Desta forma, o "gap" largo, da ordem de 4,7 eV, desfavorece a condução elétrica, o que potencializa sua utilização como isolante na eletrônica.

Em se tratando dos nanocones, a densidade de estados (Figura 6) nos mostra resultados um pouco diferentes das estruturas planas. Neste caso, ao traçarmos os gráficos das densidades de estado para essas nanoestruturas podemos comparar suas respectivas propriedades eletrônicas.

Para o os nanocones de carbono, podemos perceber que os resultados se assemelham aos expressos no plano. Desta forma, estas nanoestruturas apresentam "gap" estreito, da ordem de 1 eV, o que os caracterizam como semicondutores. Não observamos diferenças significativas entre os "gaps" dos nanocones de tamanhos diferentes.

Além de serem caracterizados como semicondutores, os nanocones de carbono também apresentam excelentes propriedades de emissão de campo, fato decorrente do elétron desemparelhado localizado no vértice do cone, quando da criação do pentágono (Li et al. 2005).

Para os nanocones de h-BN, há uma diferença mais expressiva nas densidades de estado. Apesar de ambos introduzirem estados no "gap", quando comparados ao plano de h-BN, o nanocone do tipo BNB apresenta uma energia de "gap" da ordem de 1,3 eV, o que o caracteriza como um semicondutor, já o nanocone do tipo BNN exibe um "gap" maior, aproximadamente 2,4 eV, o que inviabiliza a condução eletrônica.

\section{Conclusões}

Os resultados nos mostram que a depender da organização atômica na rede cristalina podemos obter comportamentos diferentes no que diz respeito à energia de formação e à densidade de estados. Assim, os dados obtidos nesta pesquisa se alinham ao comportamento experimental e enfatizam as inquietações sobre a manipulação da matéria a nível atômico e molecular, principalmente no tocante à aplicação destes nanomateriais em dispositivos eletrônicos de alta performance.

As implicações decorrentes desta investigação revelam que os nanomateriais, tanto derivados do carbono, quanto do h-BN, trazem outras possibilidades, para além da eletrônica que conhecemos atualmente, baseada em sua maioria no silício.

Quanto aos planos, a investigação ora realizada condiz com as pesquisas encontradas na literatura, ou seja, o grafeno apresenta características próprias de um semicondutor, enquanto o plano de h-BN apresenta-se como um isolante.

Os nanocones, de modo geral, apresentaram características diferentes uns dos outros, quanto aos seus respectivos arranjos atômicos. No caso dos nanocones de carbono, foi possível observar que a estrutura com maior número de átomos se mostrou mais estável e com um "gap" relativamente menor. Além dessas características, estes nanomateriais possuem uma concentração de cargas no vértice, que viabiliza sua empregabilidade, por exemplo, em dispositivos que utilizem emissão de elétrons em seu funcionamento.

As nanoestruturas cônicas formadas por h-BN apresentaram estabilidade similar, no entanto, a partir de suas respectivas densidades de estados foi possível observar que o BNB comporta-se como um semicondutor, enquanto o BNN como um isolante.

Para além da empregabilidade destes nanomateriais, vale ressaltar que as investigações ora realizadas contribuem para a formação de sujeitos que sejam capazes de discutir a N\&N de forma mais acurada, possibilitando inclusive que esta discussão, após um adequado processo de transposição didática, seja levada à Educação Básica, contribuindo assim com a divulgação do conhecimento científico e tecnológico. 


\section{Agradecimentos}

Agradecemos à Universidade Federal de Campina Grande (UFCG) e ao Grupo de Estrutura Eletrônica de Nanomateriais da UFPB pelo suporte e interação concedidos durante a elaboração destas pesquisas. Aos avaliadores anônimos pela revisão crítica do manuscrito. $\mathrm{O}$ presente trabalho foi realizado com apoio do CNPq, Conselho Nacional de Desenvolvimento Científico e Tecnológico- Brasil.

\section{Referências}

Alves O.L. (2004) Nanotecnologia, nanociência e nanomateriais: quando a distância entre o presente e o futuro não é apenas quentão de tempo. Parceria Estratégicas, 18: 23-40.

Azevedo S. (2006) Energetic stability of B-C-N monolayer. Physics Letters A, 351: 109-112. doi: 10.1016/j.physleta.2005.10.053

Azevedo S., Kaschny J.R., Castilho C.M.C. \& Mota F.B. (2007) A theoretical investigation of defects in a boron nitride monolayer. Nanotechnology, 18: 01-04. doi: 10.1088/09574484/18/49/495707

Cano-Marquez A.G., Schmidtw G., Ribeiro-Soares J., Cançado L.G., Rodrigues W.N., Santos A.P., Furtado C.A., Autreto P.A.S., Paupitz R., Galvão D.S. \& Jorio A. (2015) Enhanced Mechanical Stability of Gold Nanotips through Carbon Nanocone Encapsulation. Scientific Reports, 5: 01-05. doi: 10.1038/srep10408

Dantas M., Bernardo B.L. \& Azevedo S. (2015) First-principle calculations on the effect of impurities on different stacking of h-BN bilayers. Appliede Physics A, 119: 697-705. doi: 10.1007/s00339-015-9015-5

Feynman R.P. (1959) There's plenty of room at the bottom. Disponível em: http://www.its.caltech.edu/ feynman/plenty.html (Acessado em 20.04.2016).

Ge M. \& Sattler K. (1994) Observation of fullerene cones. Chemical Physics Letters, 220: 192196. doi: 10.1016/0009-2614(94)00167-7

Geim A.K. \& Novoselov K.S. (2007) The Rise of Graphene. Nature Materials, 6: 183-191. doi: 10.1038/nmat1849

Han W., Bando Y., Kurashina K. \& Sato T. (1998) Synthesis of boron nitride nanotubes from carbon nanotubes by a substitution reaction. Appliede Physics Letters, 73: 3085-3087. doi: $10.1063 / 1.122680$

Hett A. (2004) Nanotechnology: Small matter, many unknowns. Zurich: Swiss Re. 56 p.

Hohenberg P. \& Kohn W. (1964) Inhomogeneous electron gas. Physical Review, 136: 864-871. doi: 10.1103/PhysRev.136.B864

Iijima S., Ichihashi T. \& Ando Y. (1992) Pentagons, heptagons and negative curvature in graphite microtubule growth. Nature, 356: 776-778. doi: 10.1038/356776a0

Kittel C. (2013) Introdução à Física do Estado Sólido. Rio de Janeiro: LTC Editora. 578 p.

Klein A., Tiefenbacher S., Eyert V., Pettenkofer C. \& Jaegermann W. (2001) Electronic band structure of single-crystal and single-layer WS2: Influence of interlayer van der Waals interactions. Physical Review B, 64: 1-14. doi: 10.1103/PhysRevB.64.205416

Kohn W. \& Sham L.J. (1965) Self-Consistent Equations Including Exchange and Correlation Effects. Physical Review, 140: 1133-1138. doi: 10.1103/PhysRev.140.A1133

Landau L.D. (1937) Zur Theorie der phasenumwandlungen II. Physikalische Zeitschrift der Sowjetunion, 11: 26-35.

Li J.J., Gu C.Z., Wang Q., Xu P., Wang Z.L., Xu Z. \& Bai X.D. (2005) Field emission from high aspect ratio tubular carbon cones grown on gold wire. Appliede Physics Letters, 87: 01-03. doi: $10.1063 / 1.2081127$

Lopes M.D., Azevedo S., Morais F. \& Machado M. (2014) Theoretical study of carbon double cones. European Physical Journal B, 88: 01-06. doi: 10.1140/epjb/e2014-50618-x 
Novoselov K.S., Geim A.K., Morozov S.V., Jiang D., Zhang Y., Dubonos S.V., Grigorieva I.V. \& Firsov A.A. (2004) Electric Field Effect in Atomically Thin Carbon Films. Science, 306: 666669. doi: 10.1126/science.1102896

Pedreira D.O. (2011) Efeito do campo elétrico em nanocones formados por AlN e BN um estudo por primeiros princípios. Dissertação de Mestrado, Programa de Pós-Graduação em Física. Universidade Federal do Rio Grande do Norte, Natal, Rio Grande do Norte.

Rezende S.M. (2015) Materiais e Dispositivos Eletrônicos. $4^{\circ}$ edição. São Paulo: Editora Livraria da Física. $440 \mathrm{p}$.

Rubio A., Corkill J.L. \& Cohen M.L. (1994) Theory of graphitic boron nitride nanotubes. Physical Review B, 49: 5081-5084. doi: 10.1103/PhysRevB.49.5081

Schulz P.A.B. (2005) O que é Nanociência e para que serve a Nanotecnologia? Física na Escola, 6: 58-62.

Soler J.M., Artacho E., Gale J.D., Garcia A., Junquera J., Ordejon P. \& Sanchez-Portal D. (2002) The SIESTA method for ab initio order-N materials simulation. Journal of Physics: Condensed Matter, 14: 2745-2779. doi: 10.1088/0953-8984/14/11/302

Tang H. \& Ismail-Beigi S. (2007) Novel Precursors for Boron Nanotubes: The Competition of Two-Center and Three-Center Bonding in Boron Sheets. Physical Review Letters, 99: 01-04. doi: 10.1103/PhysRevLett.99.115501 